\title{
Comment on "Evolution of wall shear stress with Reynolds number in fully developed turbulent channel flow experiments"
}

\author{
R. Örlü $\odot^{*}$ and P. Schlatter $\overbrace{}^{\dagger}$ \\ SimEx/FLOW, Engineering Mechanics, KTH Royal Institute of Technology, SE-100 44 Stockholm, Sweden
}

(Received 6 August 2019; accepted 3 November 2020;

published 30 December 2020)

\begin{abstract}
The recent study by Gubian et al. [Phys. Rev. Fluids 4, 074606 (2019)], based on a new wall-shear-stress sensor in a low-Reynolds-number Re turbulent channel flow, came to the surprising conclusion that the magnitude of the fluctuating wall-shear stress $\tau_{w, r m s}^{+}$ reaches an asymptotic value of 0.44 beyond the friction Reynolds number $\operatorname{Re}_{\tau} \approx 600$. This statement is at odds with results from well-established direct numerical simulation (DNS) results that exceed the authors' highest Reynolds number by up to a factor of 5 while exhibiting a clear Reynolds-number dependence. Furthermore, they claim that "prior estimates of these quantities did not resolve the full range of wall-shear-stress fluctuations, which extended beyond 10 standard deviations above the mean." This contradicts highquality DNS results and calls for a more in-depth explanation, which is given in the present Comment. We shows that the measurements by Gubian et al. suffer from spatial-resolution issues among others, which when accounted for invalidate the statements made of an asymptotic state at $\operatorname{Re}_{\tau} \approx 600$ and resurrects the Reynolds-number dependence of $\tau_{w, r m s}^{+}$ for which DNS evidence exists exceeding $\operatorname{Re}_{\tau} \approx 600$ by an order of magnitude.
\end{abstract}

DOI: 10.1103/PhysRevFluids.5.127601

\section{BACKGROUND}

Temporally and spatially well-resolved fluctuating wall-shear-stress $\tau_{w}$ measurements are hampered by a number of complications and problems [1]. Classically, the magnitude of the fluctuating wall-shear stress, i.e., $\tau_{w, r m s}^{+}=\tau_{w, r m s} /\left\langle\tau_{w}\right\rangle$, has been indirectly inferred through measurements using hot-wire anemometry [2,3] or laser-Doppler velocimetry [4] (and more recently through particle image velocimetry (PIV) and particle tracking velocimetry (PTV), e.g., $\mu$ PIV/PTV [5] and high-speed PIV [6,7]) in the immediate vicinity of the wall exploiting the (assumed) self-similarity of the viscous sublayer [8,9]. Flush-mounted thermal-anemometry sensors have classically avoided such assumptions, but suffered instead from temporal and spatial resolution issues [2]. More recently, novel measurement techniques have been developed for this particular purpose [10-14], of which some have even been able to fully resolve the wall-shear-stress fluctuations at least for the lower-Reynolds-number range [15] (see Ref. [16] for a recent review).

The aforementioned limitations of state-of-the-art measurement techniques within the viscous sublayer of wall-bounded turbulent flows explain why most studies on the fluctuating wall-shear

\footnotetext{
*ramis@mech.kth.se

†pschlatt@mech.kth.se
}

Published by the American Physical Society under the terms of the Creative Commons Attribution 4.0 International license. Further distribution of this work must maintain attribution to the author(s) and the published article's title, journal citation, and DOI. Funded by Bibsam. 
stress rely on direct numerical simulation (DNS) data sets. Compelling evidence from these DNS data sets has over the past decade established a clear Reynolds-number Re dependence of $\tau_{w, r m s}^{+}[8,17-20]$, which, as evident, for example, from two-dimensional spectral maps of $\tau_{w}[18,21]$, is a result of the footprints of the outer-layer structures on the near-wall region [22]. These findings go along with the established failure of inner scaling for the Reynolds normal stresses and the Re dependence of the higher-order moments of the velocity fluctuations [23-29].

While the aforementioned references depict a clear picture of the Re dependence of the statistics of the fluctuating wall-shear stress and its spectral characteristics, the paper by Gubian et al. [30] presents new experimental measurements from a low-Re turbulent channel flow that combine the advantages of hot-wire anemometry and flush-mounted sensors, i.e., the measured data retain the frequency response of a hot wire while avoiding reliance on the viscous-sublayer scaling. This is accomplished through usage of a "flush-mounted, hot-wire sensor, in which the hot wire is installed over a small rectangular cavity in the sensor's base and is flush with the channel wall," thereby able to "avoid direct contact and minimize conduction from the hot wire to the sensor's substrate" [30]. Although the idea goes back to previous studies [31,32], here results on the fluctuating wall-shear-stress statistics are presented for a canonical wall-bounded turbulent flow using this technique. Note, however, that the Reynolds number in the mentioned study [30] is limited to $\operatorname{Re}_{\tau}=950$ and therefore exceeded by many existing DNS data sets.

Application of the wall-shear-stress sensor to canonical flows as done in the work by Gubian et al. [30] is hence a welcome contribution to the topic of fluctuating wall-shear-stress measurements. However, as we outline in the following, the conclusions contradict the main body of established literature data. In such a case, special care needs to be taken to ensure that the results are well substantiated. In particular, the main conclusions of their work are as follows (quotes from Ref. [30]).

(i) "Beyond $\operatorname{Re}_{\tau} \sim 600$, the wall-shear stress evolves to an asymptotic state in which the statistical moments, probability density function, and power spectra of $\tau_{w}$ become independent of Reynolds number".

(ii) "Turbulent intensity, skewness, and kurtosis of the wall-shear stress take on values that are slightly larger than those previously measured, because prior estimates of these quantities did not resolve the full range of wall-shear-stress fluctuations, which extended beyond 10 standard deviations above the mean".

(iii) An important underlying assumption of the aforementioned points is also that the authors' claim that "even though the present experimental measurements of $\tau_{w, r m s} /\left\langle\tau_{w}\right\rangle$ extend to higher friction Reynolds numbers than any others to date (to the knowledge of the authors), they show no indication of attenuation due to reduced temporal or spatial resolution as the Reynolds number increases. The constancy of $\tau_{w, r m s} /\left\langle\tau_{w}\right\rangle$ at large $\operatorname{Re}_{\tau}$ is indicative of the high-frequency response of our wall-shear-stress sensor".

\section{OVERVIEW OF CURRENT EXPERIMENTAL AND NUMERICAL OBSERVATIONS}

In the case of a new measurement technique it is common to perform tests in a well-established (canonical) flow case and to validate the results against theory or empirical evidence, thereby assessing the sensitivity and uncertainty of the new equipment. Here a wall-shear-stress sensor was used in a canonical turbulent channel flow, but the results and conclusions are at odds with the gross amount of available observations. While the authors state that "the channel and the velocity field therein have been extensively described" [30] in their previous publications, it appears that those publications are focused on scalar mixing and do not assess the Reynolds-number behavior of the mean and turbulence statistics in the near-wall region (and therefore wall-shear stress) of the channel-flow facility. Since a thorough understanding of the near-wall region of turbulent flows is very important, we believe that in the light of the statements in the mentioned paper, a more detailed analysis of the available data, literature, and experimental corrections is called for, in order to try to resolve the issues at hand. 


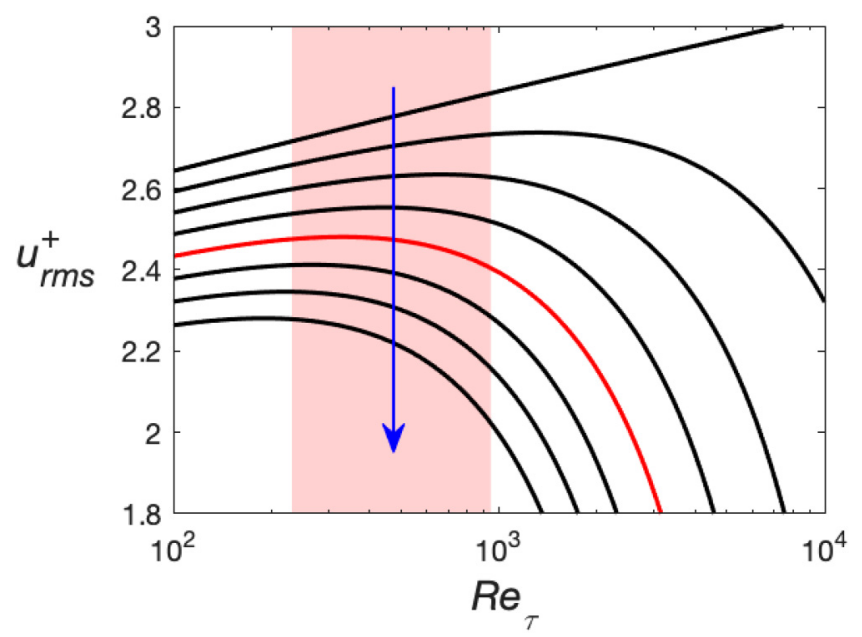

FIG. 1. Peak value of the rms value of the inner-scaled streamwise velocity fluctuations as a function of friction Reynolds number for fixed $L / \delta$ values from 0 to 0.07 (in the direction of the arrow), based on Eq. (3) in Ref. [35], where the red solid line corresponds to $L / \delta=1.25 / 30$ and the shaded area indicates the friction Reynolds-number range of the experiments of Gubian et al. [30], i.e., $\operatorname{Re}_{\tau}=230-950$.

As a starting point, it is important to state that the above-mentioned third statement, namely, that resolution effects necessarily lead to attenuation of measured fluctuations, has already been discussed most prominently by the seminal work of Hutchins et al. [33]. Indeed, it turns out that (in particular) statistics of the inner-scaled streamwise velocity fluctuations are "subject to the competing effects of the Reynolds number and $L^{+}$" [33], i.e., spatial resolution. Since there is a direct and linear relationship between the wall vorticity and the wall-shear stress, and the streamwise wallshear-stress fluctuations are related to the limiting behavior of $u_{r m s}$, i.e., $\tau_{w, r m s}^{+}=\lim _{y \rightarrow 0}\left(u_{r m s} / U\right)$, this similarly holds true at the wall. The latter, i.e., the extension to the wall, is an empirical fact based on the near constancy of the inner-scaled Kolmogorov length scale for $y^{+}<15$ [34]. In fact, as can easily be shown for an internal flow with fixed outer length scale $\delta$, a constant physical sensor length $L$ exposed to varying Reynolds numbers (e.g., centerline or bulk velocities) can measure an increasing, constant, or decreasing fluctuation amplitude depending on the Reynolds-number range and ratio of wire length and geometry $L / \delta$ (see Fig. 1 in Ref. [35]). The data from this figure are here reproduced in Fig. 1 with different values of $L / \delta$, in order to reveal how the measured fluctuation magnitude is expected to behave. As apparent from the red line, which corresponds to the $L / \delta$ ratio, i.e., hot-wire sensor length $(1.25 \mathrm{~mm})$ over the channel half-width $(30 \mathrm{~mm})$, as in the study of Gubian et al., the sensor is expected to measure a rather constant value of the rms of the fluctuating streamwise velocity fluctuations within the Reynolds-number range of the experiments by Gubian et al. (indicated through the shaded area), which is exactly what the authors observed. It is important to highlight that this trend does not imply, as the authors state, that the "sensor gives no indication of reduced spatial resolution as the Reynolds number increases" simply because of the observation that "the magnitudes of the asymptotically measured values of $\tau_{w, r m s} /\left\langle\tau_{w}\right\rangle$ show no significant reductions when the wall-unit normalized sensor length $\left(L^{+}\right)$increases with $\operatorname{Re}_{\tau}$ " [30]. Instead this observation confirms the trends depicted in Fig. 1, which clearly indicate the opposite, i.e., that the measurements are attenuated as one would anticipate from the clear (Re-dependent) increase of $L^{+}$mentioned in Table I of their paper, i.e., from 9.67 to 39.8. While Gubian et al. state these $L^{+}$values, they instead focus on the sensor areas by stating the following: "If the sensor areas normalized in wall units $\left[A^{+}=A /\left(v / u_{\tau}\right)^{2}\right]$ are calculated, then the resolution of the DNS of Ref. [18] is $\left(A^{+}=\Delta x^{+} \times \Delta z^{+}\right) 170$ to 270 , which is larger than the (projected) surface area of the wall-shear-stress sensor used herein $\left(A^{+}=L^{+} D^{+}\right)$, which is 3.2 at the highest Reynolds number 


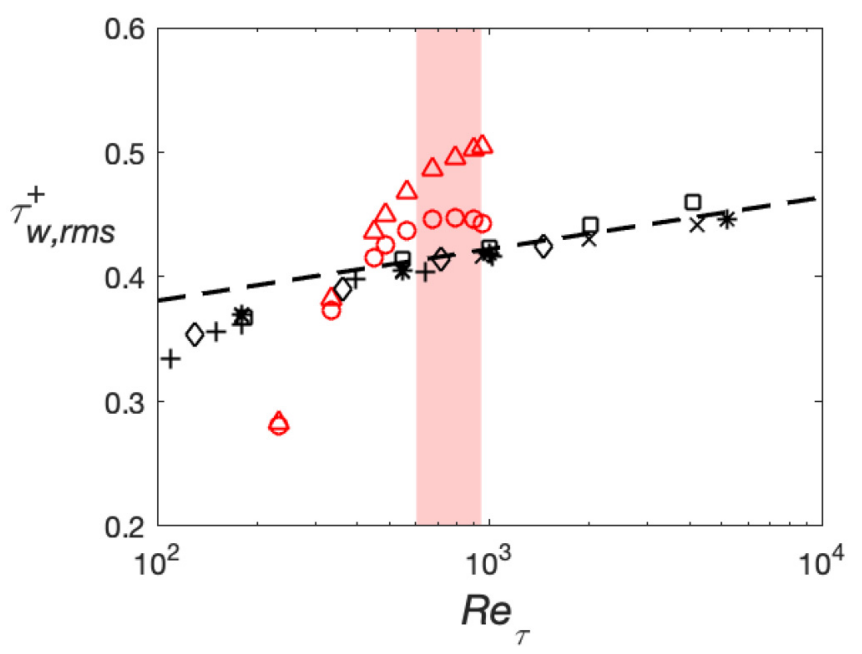

FIG. 2. Reproduction of the data from Ref. [30] (o) including its $L^{+}$corrected value ( $\triangle$ ) based on Ref. [34]. Additionally, results from channel flow DNS are shown: +, [39,40]; *, [28,41]; $\times,[42,43] ; \square,[27] ;$ and $\diamond,[17]$. The dashed line indicates the correlation for zero-pressure-gradient turbulent boundary layers [44], while the shaded area indicates the friction Reynolds-number range based on which Gubian et al. [30] concluded that $\tau_{w, r m s}^{+}$reached its asymptotic value.

studied." Ignoring the nearly order-of-magnitude overestimation of our DNS resolution [18] (see the end of this section), this comparison would lead to a nonphysical conclusion in an extreme case reasoning, viz., that an infinitely long sensor $(L \rightarrow \infty)$ combined with an infinitely thin sensor $(D \rightarrow 0)$ would fully resolve the spatial scales of the flow. As already noted, empirical evidence suggests that the attenuation behavior in the region from the wall to $y^{+}=15$ is nearly identical; hence the level of attenuation at the wall will be comparable to what is shown in Fig. 1. This can be inferred, for example, from Eq. (3.11) in Ref. [34] and other studies that investigate spatial resolution effects in thermal anemometry [36-38]. It can thus be concluded that the sensor employed in the study by Gubian et al. does suffer from spatial resolution effects, which biases the results.

Leaving the experimental evidence aside and focusing on the DNS results that have clearly established a Re dependence of the inner-peak value of the magnitude of the streamwise velocity fluctuations as well as the magnitude of the fluctuating wall-shear stress, Gubian et al. make a noteworthy observation when they compare their data to numerical results. As apparent from Fig. 2, which reproduces the data by Gubian et al., their measured data indeed exhibit a plateau (or as they call it an asymptotic state) at a level higher than any previous experimentally obtained value at comparable $\operatorname{Re}_{\tau}$ and most simulation data. This observation led them to state that their "measured asymptotic value of 0.44 is even closer to values obtained by Hu et al., Lenaers et al., and Lee and Moser, who obtained maximum values of $0.43,0.42$, and 0.45 , respectively, thus confirming the results of prior high-Reynolds-number direct numerical simulations." In this statement, the lower Reynolds number is unfortunately not taken into account. As is apparent from Fig. 2, all DNS data sets, whether they are from channel, pipe (see, e.g., Ref. [20]), or turbulent boundary layer (TBL) flows [44,45], exhibit a clear Re trend; similar compilations can be found in Refs. [12,19], but even in Fig. 1(b) of Ref. [30]. It should be noted that we have excluded DNS data sets that either exhibit transitional characteristics [46-48] or depict reminiscence from tripping or inflow effects $[49,50]$ as discussed in Refs. [18,51,52].

A minor point regarding the resolution in DNS should also be mentioned, as it has been used as a possible explanation of the apparent disagreement of the new measurements and the existing data. To make this argument Gubian et al. report mistakenly incorrect streamwise and spanwise 
grid resolutions for the DNS reported in Ref. [44]. In particular, they state that the inner-scaled streamwise and spanwise resolutions are up to 25.3 and 10.8, respectively, while they are in fact 9 and 4, respectively; we suspect that the former values are probably (mistakenly) taken from our first DNS of a TBL [53] and large-eddy simulation [54]. As investigated in detail in Ref. [24] as well as reported by Gubian et al., these resolutions are clearly sufficient to resolve the wall-shear-stress fluctuations and the speculations offered about an insufficient streamwise resolution in previous DNS are unsubstantiated with respect to the Reynolds-number scaling of the magnitude of the fluctuating wall-shear stress $\tau_{w, r m s}^{+}$, since the low-frequency content (i.e., large-scale structures) of the fluctuations away from the wall was found to be responsible for the increase in magnitude $[18,25]$.

\section{POTENTIAL RESOLUTION}

Based on the arguments provided so far, it might be interesting to explore how standard spatial resolution $\left(L^{+}\right)$correction schemes would affect the fluctuating wall-shear-stress measurements. Note that, in the following, we do not attempt to explain the observed discrepancies with literature data as there is not enough information, e.g., about the calibration procedure, available to attempt this. This section is merely meant as an illustration of how spatial resolution effects can bias the trends within a limited Reynolds-number range. We thus proceed to "correct" the measured $\tau_{w, r m s}^{+}$ values of Gubian et al. in order to see whether the apparent asymptotic state the authors seemingly observe beyond a $\operatorname{Re}_{\tau}$ of 600 is an artifact of the ignored spatial resolution effects of their sensor. Utilizing the well-established correction scheme by Smits et al. [34] on the measured $\tau_{w, r m s}^{+}$values (by setting $y^{+}=0$ in their Eq. (3.11)), we obtain even higher values for $\tau_{w, r m s}^{+}$as apparent from Fig. 2 (upward triangles). However, at the same time the Re trend of $\tau_{w, r m s}^{+}$is revealed, which was previously obscured by $L^{+}$effects. It should be noted that any other correction scheme could have been used to recover the revealed Reynolds-number trend $[37,55,56]$ since they are calibrated against DNS data within the viscous sublayer. While the Re trend is recovered, it is clear that the amplitudes and the Re dependence are too high and strong, respectively, compared to existing correlations values. This indicates that there are other effects that might need to be accounted for; however, this would remain speculative without further insight into the calibration process of the sensor.

One reason for the aforementioned overcorrection of $\tau_{w, r m s}^{+}$is that it assumes that spatial resolution effects are the only deficiency for the developed sensor. However, another potential error source could be the lack of a dynamic calibration as usually performed for new (wall-shear-stress) sensors (see, e.g., Refs. [57,58]). While the authors state that "the small (1-mm-wide by 0.1-mm-deep) cavity is to avoid direct contact and minimize conduction from the hot wire to the sensors substrate, which greatly increases the sensor's frequency response $(>10 \mathrm{kHz})$," such an estimate about a particular dynamic response is difficult to give in the absence of a direct characterization of the dynamic response [57].

\section{SUMMARY AND CONCLUSIONS}

The recent work by Gubian et al. based on new measurements made strong statements with regard to the scaling of the fluctuating wall-shear stress and in particular our previous work on this subject [18]. As a main result it was stated that an asymptotic value for $\tau_{w, r m s}^{+}$(and related turbulence statistics) would be reached at $\operatorname{Re}_{\tau} \approx 600$. This is in disagreement with existing high-quality DNS from turbulent channel, pipe, and boundary-layer flows (including data that exceed the experiments in terms of Re by a factor of up to 5) which exhibit a clear increasing Re trend. Furthermore, claims were made that their new sensor exhibits "high-spatial and high-temporal resolution," while in the present Comment we argue that (i) their sensor exhibits strong effects of insufficient spatial resolution, which obscures the Re trend, (ii) when corrected for spatial resolution effects the Re dependence of $\tau_{w, r m s}^{+}$is restored, and (iii) the claimed high temporal resolution and conclusions 
from the new sensor, without a direct characterization of the dynamic response, is not confidently demonstrated.

It should be noted that we abstain from speculations on why the recovered amplitudes and Re trends of $\tau_{w, r m s}^{+}$are too large and strong, respectively, once compensated for spatial resolution effects compared to the literature. However, it might be worth investigating the static and dynamic calibration procedure, which will affect not only the mean value $\left\langle\tau_{w}\right\rangle$ but also $\tau_{w, r m s}^{+}$, which is scaled by the mean. The authors calibrated their flush-mounted wall-shear-stress sensor in "steady, laminar, fully developed flows in a high-aspect-ratio, rectangular test section" [59]. However, when it comes to wall-bounded turbulent flow measurements, wall-mounted sensors are often calibrated in situ, i.e., submerged in the near-wall region of a turbulent flow (see, e.g., Refs. $[2,3,60,61]$ ).

At the same time, the present Comment should not overshadow the advantages of the new wall-shear-stress sensor presented by Gubian et al., viz., its ability to minimize conduction effects, which has, in combination with a shorter sensing element, potential for future wall-shear-stress measurements even in more complex geometries. This route is certainly worth pursuing, especially in the absence of alternative measurement techniques, and a dynamic calibration as outlined, for example, in Ref. [57] would contribute to clear up many of the open questions that are discussed in Ref. [30] and herein.

\section{ACKNOWLEDGMENT}

Financial support provided by the Knut and Alice Wallenberg Foundation is gratefully acknowledged.

[1] R. Vinuesa and R. Örlü, in Experimental Aerodynamics, edited by S. Discetti and A. Ianiro (CRC Press, Boca Raton, 2017), pp. 393-428.

[2] P. H. Alfredsson, A. V. Johansson, J. H. Haritonidis, and H. Eckelmann, The fluctuating wall-shear stress and the velocity field in the viscous sublayer, Phys. Fluids 31, 1026 (1988).

[3] J.-D. Rüedi, H. M. Nagib, J. M. Österlund, and P. A. Monkewitz, Unsteady wall-shear measurements in turbulent boundary layers using MEMS, Exp. Fluids 36, 393 (2004).

[4] F. Durst, J. Jovanovic, and J. Sender, LDA measurements in the near-wall region of a turbulent pipe flow, J. Fluid Mech. 295, 305 (1995).

[5] W. Li, D. Roggenkamp, W. Jessen, M. Klaas, and W. Schröder, Reynolds number effects on the fluctuating velocity distribution in wall-bounded shear layers, Meas. Sci. Technol. 28, 015302 (2016).

[6] C. E. Willert, High-speed particle image velocimetry for the efficient measurement of turbulence statistics, Exp. Fluids 56, 17 (2015).

[7] C. E. Willert, C. Cuvier, J. M. Foucaut, J. Klinner, M. Stanislas, J.-P. Laval, S. Srinivasan, J. Soria, O. Amili, C. Atkinson, C. J. Kähler, S. Scharnowski, R. Hain, A. Schröder, R. Geisler, J. Agocs, and A. Rose, Experimental evidence of near-wall reverse flow events in a zero pressure gradient turbulent boundary layer, Exp. Therm. Fluid Sci. 91, 320 (2018).

[8] P. H. Alfredsson, R. Örlü, and P. Schlatter, The viscous sublayer revisited-exploiting self-similarity to determine the wall position and friction velocity, Exp. Fluids 51, 271 (2011).

[9] R. Örlü, J. H. M. Fransson, and P. H. Alfredsson, On near wall measurements of wall bounded flows-The necessity of an accurate determination of the wall position, Prog. Aerosp. Sci. 46, 353 (2010).

[10] S. Große and W. Schröder, Dynamic wall-shear stress measurements in turbulent pipe flow using the micro-pillar sensor MPS ${ }^{3}$, Int. J. Heat Fluid Flow 29, 830 (2008).

[11] S. Große and W. Schröder, High Reynolds number turbulent wind tunnel boundary layer wall-shear stress sensor, J. Turbul. 10, N1 (2009).

[12] L. Keirsbulck, L. Labraga, and M. Gad-el-Hak, Statistical properties of wall shear stress fluctuations in turbulent channel flows, Int. J. Heat Fluid Flow 37, 1 (2012). 
[13] E. P. Gnanamanickam, B. Nottebrock, S. Große, J. P. Sullivan, and W. Schröder, Measurement of turbulent wall shear-stress using micro-pillars, Meas. Sci. Technol. 24, 124002 (2013).

[14] C Brücker, Evidence of rare backflow and skin-friction critical points in near-wall turbulence using micropillar imaging, Phys. Fluids 27, 031705 (2015).

[15] Y. Liu, M. Klaas, and W. Schröder, Measurements of the wall-shear stress distribution in turbulent channel flow using the micro-pillar shear stress sensor MPS ${ }^{3}$, Exp. Therm. Fluid Sci. 106, 171 (2019).

[16] R. Örlü and R. Vinuesa, Instantaneous wall-shear-stress measurements: Advances and application to nearwall extreme events, Meas. Sci. Technol. 31, 112001 (2020).

[17] Z. W. Hu, C. L. Morfey, and N. D. Sandham, Wall pressure and shear stress spectra from direct simulations of channel flow, AIAA J. 44, 1541 (2006).

[18] R. Örlü and P. Schlatter, On the fluctuating wall shear stress in zero pressure-gradient turbulent boundary layer flows, Phys. Fluids 23, 021704 (2011).

[19] L. Keirsbulck, G. Fourrié, L. Labraga, and M. Gad-el-Hak, Scaling of statistics in wall-bounded turbulent flows, C. R. Mec. 340, 420 (2012).

[20] G. K. El Khoury, P. Schlatter, A. Noorani, P. F. Fischer, G. Brethouwer, and A. V. Johansson, Direct numerical simulation of turbulent pipe flow at moderately high Reynolds numbers, Flow Turbul. Combust. 91, 475 (2013).

[21] G. Eitel-Amor, R. Örlü, and P. Schlatter, Simulation and validation of a spatially evolving turbulent boundary layers up to $\operatorname{Re}_{\theta}=8300$, Int. J. Heat Fluid Flow 47, 57 (2014).

[22] I. Marusic, R. Mathis, and N. Hutchins, High Reynolds number effects in wall turbulence, Int. J. Heat Fluid Flow 31, 418 (2010).

[23] R. Mathis, N. Hutchins, and I. Marusic, A predictive inner-outer model for streamwise turbulence statistics in wall-bounded flows, J. Fluid Mech. 681, 537 (2011).

[24] P. Lenaers, Q. Li, G. Brethouwer, P. Schlatter, and R. Örlü, Rare backflow and extreme wall-normal velocity fluctuations in near-wall turbulence, Phys. Fluids 24, 035110 (2012).

[25] R. Mathis, I. Marusic, S. I. Chernyshenko, and N. Hutchins, Estimating wall-shear-stress fluctuations given an outer region input, J. Fluid Mech. 715, 163 (2013).

[26] S. Pirozzoli and M. Bernardini, Probing high-Reynolds-number effects in numerical boundary layers, Phys. Fluids 25, 021704 (2013).

[27] M. Bernardini, S. Pirozzoli, and P. Orlandi, Velocity statistics in turbulent channel flow up to $\operatorname{Re}_{\tau}=4000$, J. Fluid Mech. 742, 171 (2014).

[28] M. Lee and R. D. Moser, Direct numerical simulation of turbulent channel flow up to $\operatorname{Re}_{\tau} \approx 5200$, J. Fluid Mech. 774, 395 (2015).

[29] Y. Yamamoto and Y. Tsuji, Numerical evidence of logarithmic regions in channel flow at $\operatorname{Re}_{\tau}=8000$, Phys. Rev. Fluids 3, 012602 (2018).

[30] P.-A. Gubian, J. Stoker, J. Medvescek, L. Mydlarski, and B. R. Baliga, Evolution of wall shear stress with Reynolds number in fully developed turbulent channel flow experiments, Phys. Rev. Fluids 4, 074606 (2019).

[31] P. G. Spazzini, G. Iuso, M. Onorato, and N. Zurlo, Design, test and validation of a probe for time-resolved measurement of skin friction, Meas. Sci. Technol. 10, 631 (1999).

[32] D. Sturzebecher, S. Anders, and W. Nitsche, The surface hot wire as a means of measuring mean and fluctuating wall shear stress, Exp. Fluids 31, 294 (2001).

[33] N. Hutchins, T. B. Nickels, I. Marusic, and M. S. Chong, Hot-wire spatial resolution issues in wallbounded turbulence, J. Fluid Mech. 635, 103 (2009).

[34] A. J. Smits, J. P. Monty, M. Hultmark, S. C. C. Bailey, N. Hutchins, and I. Marusic, Spatial resolution correction for wall-bounded turbulence measurements, J. Fluid Mech. 676, 41 (2011).

[35] R. Örlü and P. H. Alfredsson, On spatial resolution issues related to time-averaged quantities using hotwire anemometry, Exp. Fluids 49, 101 (2010).

[36] A. Segalini, R. Örlü, P. Schlatter, P. H. Alfredsson, J.-D. Rüedi, and A. Talamelli, A method to estimate turbulence intensity and transverse Taylor microscale in turbulent flows from spatially averaged hot-wire data, Exp. Fluids 51, 693 (2011). 
[37] M. A. Miller, B. Estejab, and S. C. C. Bailey, Evaluation of hot-wire spatial filtering corrections for wall turbulence and correction for end-conduction effects, Exp. Fluids 55, 1735 (2014).

[38] R. Örlü and R. Vinuesa, in Experimental Aerodynamics (Ref. [1]), pp. 257-303.

[39] H. Abe, H. Kawamura, and H. Choi, Very large-scale structures and their effects on the wall shear-stress fluctuations in a turbulent channel flow up to $\operatorname{Re}_{\tau}=640$, J. Fluid Eng. 126, 835 (2004).

[40] H. Abe, H. Kawamura, and Y. Matsuo, Surface heat-flux fluctuations in a turbulent channel flow up to $\operatorname{Re}_{\tau}=1020$ with $\operatorname{Pr}=0.025$ and 0.71, Int. J. Heat Fluid Flow 25, 404 (2004).

[41] R. D. Moser, J. Kim, and N. N. Mansour, Direct numerical simulation of turbulent channel flow up to $\operatorname{Re}_{\tau}=$ 590, Phys. Fluids 11, 943 (1999).

[42] J. C. Del Álamo, J. Jiménez, P. Zandonade, and R. D. Moser, Scaling of the energy spectra of turbulent channels, J. Fluid Mech. 500, 135 (2004).

[43] A. Lozano-Durán and J. Jiménez, Effect of the computational domain on direct simulations of turbulent channels up to $\operatorname{Re}_{\tau}=4200$, Phys. Fluids 26, 011702 (2014).

[44] P. Schlatter and R. Örlü, Assessment of direct numerical simulation data of turbulent boundary layers, J. Fluid Mech. 659, 116 (2010).

[45] P. Schlatter and R. Örlü, Turbulent boundary layers at moderate Reynolds numbers: Inflow length and tripping effects, J. Fluid Mech. 710, 5 (2012).

[46] X. Wu and P. Moin, Direct numerical simulation of turbulence in a nominally zero-pressure-gradient flat-plate boundary layer, J. Fluid Mech. 630, 5 (2009).

[47] X. Wu and P. Moin, Transitional and turbulent boundary layer with heat transfer, Phys. Fluids 22, 085105 (2010).

[48] X. Wu, Establishing the generality of three phenomena using a boundary layer with free-stream passing wakes, J. Fluid Mech. 664, 193 (2010).

[49] G. Khujadze and M. Oberlack, DNS and scaling laws from new symmetry groups of ZPG turbulent boundary layer flow, Theor. Comput. Fluid Dyn. 18, 391 (2004).

[50] G. Khujadze and M. Oberlack, Proceedings of the Fifth International Symposium on Turbulence and Shear Flow Phenomena, München, 2007 (Begell House, Danbury, 2007).

[51] J. Jiménez, S. Hoyas, M. P. Simens, and Y. Mizuno, Turbulent boundary layers and channels at moderate Reynolds numbers, J. Fluid Mech. 657, 335 (2010).

[52] P. Schlatter, Q. Li, R. Örlü, F Hussain, and D. S. Henningson, On the near-wall vortical structures at moderate Reynolds numbers, Eur. J. Mech. B 48, 75 (2014).

[53] P. Schlatter, R. Örlü, Q. Li, G. Brethouwer, J. H. M. Fransson, A. V. Johansson, P. H. Alfredsson, and D. S. Henningson, Turbulent boundary layers up to $\mathrm{Re}_{\theta}=2500$ studied through simulation and experiment, Phys. Fluids 21, 051702 (2009).

[54] P. Schlatter, Q. Li, G. Brethouwer, A. V. Johansson, and D. S. Henningson, Simulations of spatially evolving turbulent boundary layers up to $\operatorname{Re}_{\theta}=4300$, Int. J. Heat Fluid Flow 31, 251 (2010).

[55] C. Chin, N. Hutchins, A. S. H. Ooi, and I. Marusic, Spatial resolution correction for hot-wire anemometry in wall turbulence, Exp. Fluids 50, 1443 (2011).

[56] J. Philip, N. Hutchins, J. P. Monty, and I. Marusic, Spatial averaging of velocity measurements in wallbounded turbulence: Single hot-wires, Meas. Sci. Technol. 24, 115301 (2013).

[57] V. Chandrasekaran, A. Cain, T. Nishida, L. N. Cattafesta, and M. Sheplak, Dynamic calibration technique for thermal shear-stress sensors with mean flow, Exp. Fluids 39, 56 (2005).

[58] S. Große, T Soodt, and W. Schröder, Dynamic calibration technique for the micro-pillar shear-stress sensor MPS ${ }^{3}$, Meas. Sci. Technol. 19, 105201 (2008).

[59] S. Afara, J. Medvescek, L. Mydlarski, B. R. Baliga, and M. MacDonald, Development of a wall-shearstress sensor and measurements in mini-channels with partial blockages, Exp. Fluids 55, 1734 (2014).

[60] R. Ruderich and H. H. Fernholz, An experimental investigation of a turbulent shear flow with separation, reverse flow, and reattachment, J. Fluid Mech. 163, 283 (1986).

[61] G. J. Kunkel and I. Marusic, An approximate amplitude attenuation correction for hot-film shear stress sensors, Exp. Fluids 34, 285 (2003). 